\title{
Consumo de alcohol en la adolescencia. Consideraciones médicas y orientaciones educativas
}

\author{
Francisco David Rodríguez García, ${ }^{1}$ María Luisa Sanchiz Ruiz, ${ }^{2}$ Rafael Bisquerra Alzina ${ }^{3}$
}

Actualización por temas

\section{SUMMARY}

Alcohol is the most commonly consumed psychoactive drug among Spanish adolescents. The effects of alcohol on human brain, specifically on neurotransmission, are broadly studied. The adolescent brain is especially vulnerable to the effects of alcohol due to the intense and active processes of synapses restructuring ocurring during this period. To achieve and establish educational interventions directed to facilitate and eradicate harmful habits related to alcohol consumption during adolescence, it is necessary to join resources, knowledge and forces focused on a better understanding of the biological effects of alcohol and the harm produced in the emotional, social and familiar realms. Here, we discuss that intervention needs previous evaluation and should focus on Secondary Education, a crucial period in human development.

Key words: Alcohol, adolescence, brain, educational counseling.

\section{RESUMEN}

El consumo de alcohol por parte de los adolescentes supera en España el consumo de otras sustancias psicoactivas. Los daños que el cerebro humano sufre a causa del etanol, especialmente en la etapa de la adolescencia, son objeto de muchos estudios y se centran principalmente en cómo se afecta la neurotransmisión. Además, la vulnerabilidad del cerebro de los adolescentes a la influencia del alcohol ofrece rasgos peculiares por cuanto se encuentra en una etapa de intensa actividad de remodelación sináptica. Es necesario unir fuerzas, conocimientos y recursos dirigidos a un mejor conocimiento, tanto de los efectos biológicos del alcohol en el individuo adolescente como de los derivados del consumo en los ámbitos emocional, social y familiar, para diseñar actuaciones educativas que faciliten la modificación o erradicación de hábitos no saludables relacionados con la ingesta de alcohol. La evaluación previa se dirige a promover la calidad de vida en la Educación Secundaria, una etapa crucial en el desarrollo global del ser humano.

Palabras clave: Alcohol, adolescencia, cerebro, asesoría educativa.

\section{ADOLESCENCIA Y CONSUMO DE ALCOHOL}

Según la Organización Mundial de la Salud, la adolescencia es el periodo de edad comprendido entre los 10 y los 19 años (la segunda década). ${ }^{1}$ Esta etapa, a su vez, se subdivide en dos grupos de edad: de 10-14 años (adolescencia temprana) y de 15-19 años (adolescencia tardía). ${ }^{2}$ La adolescencia es una etapa de cambios sustanciales en tiempos cortos, que afectan al desarrollo y la consolidación de las funciones del organismo. Las transformaciones incluyen la aparición de la pubertad, el afianzamiento y la consolidación de las relaciones sociales con los iguales o el forcejeo por conseguir la in- dependencia respecto de los padres o tutores. ${ }^{3,4}$ El tiempo y la forma de la adolescencia son cambiantes. El comienzo de la pubertad tiende a ser más temprano, mientras que se retrasa la edad en la que se consiguen papeles sociales más estables. ${ }^{2}$ Esta etapa de la vida se caracteriza por un desarrollo muy importante del cerebro que incluye el establecimiento, la remodelación y la consolidación de los circuitos neuronales en lugares clave de la corteza prefrontal y en otras áreas corticales y subcorticales, esenciales en las funciones ejecutivas del cerebro. ${ }^{5}$

Consumir alcohol no es un problema exclusivo de la adolescencia, pues compete a toda la población. Pero el comienzo del consumo suele producirse durante esta etapa.

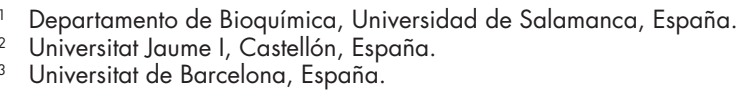


Por tanto, si dirigimos la atención, el análisis y la acción a los adolescentes, podemos evitar daños prematuros y futuros, al tiempo que sometemos a una revisión crítica ciertas actitudes y conductas de los adultos que estimulan y promueven el consumo.

En su entorno habitual, los adolescentes encuentran mensajes ambiguos. ${ }^{6}$ Por un lado, desde diversas instancias públicas y privadas se denuncian los efectos nocivos del alcohol y, por otro, se estimula el consumo con campañas publicitarias de las empresas vendedoras y distribuidoras, que a menudo incluyen términos engañosos (v.gr., consumo "responsable" y "moderado") y presentan los mensajes asociados a imágenes de libertad y diversión. Los efectos derivados del consumo de alcohol no constituyen sólo un problema individual, sino también comunitario. Aunque el riesgo se define como una acción que puede implicar una pérdida, cada sujeto lo entiende en función de sus propias percepciones. ${ }^{7}$

En las patologías asociadas al consumo de alcohol ${ }^{8}$ son destacables los trastornos relacionados con el comportamiento hacia el alcohol (abuso o consumo perjudicial y dependencia del alcohol) y las alteraciones relacionadas con los efectos directos sobre el cerebro (intoxicación aguda, abstinencia alcohólica, delirium, trastorno amnésico, síndrome de Wernicke-Korsakow y demencia, trastornos psicóticos, trastornos del estado de ánimo, trastornos de ansiedad, disfunciones sexuales y trastornos del sueño). Sin embargo, guiados principalmente por el placer momentáneo asociado a la bebida, forzados por la presión del grupo de amigos, impulsados por sus deseos de mejorar la comunicación con los demás o como forma de evasión, los humanos obvian en gran medida los daños asociados al consumo.

En España, se realizan diversos estudios para conocer en qué medida consumen drogas los adolescentes. Entre ellos podemos destacar la encuesta estatal sobre uso de drogas en estudiantes de secundaria (ESTUDES), que cubre todo el territorio español y se centra en la adolescencia tardía. Los datos más recientes se refieren a ESTUDES $2010^{\circ}$ (Muestra de 31967 estudiantes, entre 14 y 18 años). En ella se señala que la sustancia psicoactiva de consumo más generalizado entre los encuestados es el alcohol. El 73.6\% ha tomado bebidas alcohólicas en los últimos doce meses. El 63\% ha bebido en los últimos 30 días previos a la encuesta. Un 36.7\% de la muestra ha bebido en forma de atracón (binge drinking) en los últimos 30 días previos a la encuesta. La bebida en forma de atracón consiste en beber unos $50 \mathrm{~g}$ de alcohol en un corto periodo de tiempo, 2-3 horas, al menos una vez a la semana. ESTUDES establece la edad media de inicio en el consumo de alcohol en los 13.7 años. Por su parte, la prevalencia del consumo de alcohol en la población señalada se mantiene prácticamente constante desde 1994.

La expresión más paradigmática del consumo en atracón la encontramos en los denominados "botellones" y "macrobotellones",10,11 reuniones multitudinarias, celebradas generalmente al aire libre y durante los fines de semana, de sujetos adolescentes y jóvenes, principalmente. También se observa esta conducta en adultos (generalmente, en otros escenarios). El consumo intensivo y repetido supone un estímulo para el cerebro que incluye el aumento rápido de la concentración de alcohol en sangre, su ausencia durante el tiempo de abstinencia. España pertenece a la Unión Europea (UE), la región del mundo en la que más de un quinto de la población de más de 15 años consume alcohol de forma episódica e intensa (binge drinking). ${ }^{12}$

\section{ALCOHOL Y NEUROTRANSMISIÓN}

El alcohol etílico es una sustancia exógena que el organismo metaboliza y transforma en compuestos asimilables o desechables. Su estructura y propiedades químicas le permiten llegar a todos los órganos y tejidos una vez absorbido. El etanol atraviesa las membranas de las células e interacciona con todos los elementos que las componen. Se ha descrito el efecto específico del etanol sobre proteínas receptoras y conductos para iones. ${ }^{13}$ Por ejemplo, receptores NMDA (N-Metil_D-Aspartato) para glutamato, receptores para glicina, receptores para la acetilcolina (tipo nicotínico), receptores para serotonina (tipo 5HT3), conductos para calcio (tipo L) y algunos conductos para el potasio. ${ }^{14}$ La acción del etanol en estas proteínas es detectable a concentraciones a partir de $1 \mathrm{mM}(46 \mathrm{mg} / \mathrm{L})$. La interferencia del etanol con los sistemas de neurotransmisión es la base del daño que el alcohol provoca, a corto y a largo plazos, al cerebro. La interacción del etanol con las proteínas mencionadas es dosis-dependiente y responsable de efectos agudos como la desinhibición, la sedación o el sueño. Los efectos del etanol se diversifican para perturbar otros sistemas neurotransmisores, opioides, dopamina y endocanabinoides, que se relacionan con mecanismos de refuerzo positivo y recompensa y sirven de punto de inicio de la dependencia alcohólica. Una vez consolidada la dependencia, intervienen otros sistemas neurotransmisores (la hormona liberadora de corticotropina, o CRH, y el neuropéptido Y, NPY) que tienen una estrecha relación con la activación patológica de los circuitos que controlan el estrés y los estados de emergencia. ${ }^{15}$

Los estudios de mapas de densidad de la sustancia gris señalan que no todas las estructuras del cerebro maduran al mismo ritmo y tiempo. ${ }^{16}$ Las diferencias en maduración cerebral pueden explicar, en parte, diferencias de sensibilidad a los efectos del etanol en las etapas de la vida. En la adolescencia, por ejemplo, los efectos placenteros del alcohol aparecen a dosis muy bajas, mientras que los efectos desagradables, ligados a la intoxicación, emergen a dosis más elevadas. Este fenómeno, relacionado con la sensibilidad del individuo a los efectos del etanol, estimula y refuerza la conducta bebedora en los adolescentes. ${ }^{17}$

Se ha demostrado una clara correlación entre la edad en la que se comienza a consumir alcohol y el riesgo de ser 
alcohólico en la edad adulta. Cuanto antes se comienza a beber, el riesgo es mayor. ${ }^{18}$ Los programas de prevención primaria o secundaria, que ayuden a los adolescentes a tomar conciencia de los efectos dañinos de esta droga y a desarrollar habilidades de autocontrol y toma de decisiones, constituyen un enfoque relevante.

Debido a restricciones legales y éticas, no es factible realizar ciertos estudios acerca de los efectos del alcohol en adolescentes humanos. Por ello, se llevan a cabo ensayos que utilizan modelos animales, fundamentalmente roedores $^{19-22}$ o modelos in vitro. ${ }^{23}$ La investigación básica en estos modelos pretende explicar los mecanismos celulares y moleculares que subyacen a la etiología y que son responsables de las consecuencias del consumo de alcohol. ${ }^{4}$

\section{Políticas de prevención}

Las políticas de prevención incluyen normas y leyes que establecen las autoridades competentes, así como programas educativos en diversos contextos y la educación en el entorno familiar. Según el informe de 2009 de la Organización Mundial de la Salud sobre las intervenciones dirigidas a la reducción del daño producido por el alcohol,12 las políticas de información y educación, aisladas, tienen un escaso impacto en la conducta bebedora de los individuos en general. Sin embargo, tienen sentido en el mantenimiento y enriquecimiento de una conciencia social e individual sobre el consumo de alcohol y sus consecuencias. Son acuciantes acciones multidisciplinares que incluyan políticas relacionadas con el precio, la prohibición de consumo y venta en determinados lugares y a menores de edad, así como acciones de formación y desarrollo integral en los distintos ámbitos de la comunidad en la que el sujeto desarrolla su actividad.

Las agendas y los programas de salud de diferentes organismos e instancias deben situar el foco en la adolescencia para llevar a cabo políticas de mejora de la salud durante esa etapa y la vida futura. ${ }^{2}$ No se trata de mirar la adolescencia con paternalismo y condescendencia, a veces con cierto desprecio. La adolescencia es una etapa de crecimiento, de posibilidades, de ajustes, que reclama atención en todos los ámbitos del desarrollo del individuo y la promoción de su salud.

\section{Orientaciones educativas}

Es importante plantear soluciones y orientaciones educativas que ayuden a los adolescentes y se hagan extensivas a familiares, educadores y a la comunidad en general. Recurrir a sustancias psicoactivas no es la solución. ${ }^{24}$

Desde la psicopedagogía entendemos la orientación como un proceso continuo que debe ayudar al pleno desarrollo del individuo y a capacitarle para la autorientación y la participación activa, crítica y transformadora de la sociedad en la que vive. ${ }^{25}$ Ante el consumo de alcohol por parte de la población adolescente, consideramos importante la puesta en marcha de programas preventivos y de intervención que, con una visión holística, tengan en cuenta todos los agentes implicados y capaciten a los adolescentes para ser conscientes de la responsabilidad que tienen en su propio desarrollo.

Para que las propuestas educativas se ajusten a la población a la que se dirigen, es importante realizar un análisis adecuado y determinar las demandas y necesidades de los destinatarios. En ocasiones, existe la necesidad pero no se es consciente de ella y, por tanto, no se ha formulado la demanda; de ahí la importancia de concienciar a los implicados. No ajustarse a este primer requisito puede poner en peligro el mejor de los programas o reducir su efectividad. Los destinatarios del programa deben percibir la necesidad del mismo, hacer suya la demanda, para que aquél no sea percibido como algo impuesto desde fuera, sino como algo solicitado y deseado.

\section{Destinatarios de los programas. Tipología}

Hay que tener en cuenta que no todos los individuos tienen el mismo grado de acercamiento al alcohol. Pantoja y Añanos $^{26}$ diferencian cuatro grupos:

a) Grupo de la normalidad, compuesto por menores que no consumen o cuyo consumo es esporádico.

b) Grupo de riesgo, menores que se han iniciado en el uso y tienen un riesgo elevado de convertirse en drogodependientes.

c) Grupo crítico-dependiente, semejante al anterior pero con un consumo habitual; se acercan a la exclusión.

d) Grupo excluido, menores inmersos en el mundo de la droga, ubicados de lleno en el mundo de la exclusión ("niños y niñas de la calle").

Por su parte, Arbex ${ }^{24}$ distingue cinco grupos:

a) Menores abstemios o consumidores moderados.

b) Menores consumidores abusivos de alcohol los fines de semana.

c) Menores bebedores abusivos de alcohol los fines de semana, incluyendo consumo de hachís.

d) Menores consumidores de alcohol, hachís y consumo esporádico de otras sustancias.

e) Menores con un consumo avanzado de drogas.

En función de la clasificación que establezcamos, elegiremos el programa más adecuado.

\section{TIPOS DE PROGRAMA}

Uno de los objetivos de la educación es desarrollar la prevención, desde la etapa infantil hasta la edad adulta, y, en relación con el consumo de alcohol, haciendo un especial hincapié en la educación secundaria (14-18 años). 
Cuadro 1. Tipo de prevención y programas según destinatarios

\begin{tabular}{|c|c|c|}
\hline $\begin{array}{l}\text { Tipo } \\
\text { de prevención }\end{array}$ & Modalidad & Destinatarios \\
\hline Primaria & $\begin{array}{l}\text { Prevención } \\
\text { universal }\end{array}$ & $\begin{array}{l}\text { Grupo de la normalidad. } \\
\text { Menores abstemios o consumidores } \\
\text { esporádicos. }\end{array}$ \\
\hline Secundaria & $\begin{array}{l}\text { Prevención } \\
\text { selectiva }\end{array}$ & $\begin{array}{l}\text { Grupo de riesgo. } \\
\text { Grupo crítico-dependiente. } \\
\text { Menores consumidores abusivos de } \\
\text { alcohol los fines de semana y consu- } \\
\text { midores de alcohol + hachís/ + otras } \\
\text { sustancias. }\end{array}$ \\
\hline Terciaria & $\begin{array}{l}\text { Prevención } \\
\text { indicada }\end{array}$ & $\begin{array}{l}\text { Grupo excluido. } \\
\text { Menores con un consumo avanzado } \\
\text { de drogas. }\end{array}$ \\
\hline
\end{tabular}
ción: ${ }^{27}$

Podemos distinguir los siguientes tipos de preven-

1. Prevención primaria: se ofrece antes de que surja el problema.

2. Prevención secundaria: actúa en cuanto aparece el problema.

3. Prevención terciaria: actúa ofreciendo tratamiento y rehabilitación cuando el problema está ampliamente desarrollado.

En el cuadro 1 conjugamos el tipo de prevención con la modalidad de programa adaptado a la situación de los destinatarios, tomando como referencia las clasificaciones realizadas por Pantoja y Añanos ${ }^{26}$ y Árbex. ${ }^{24}$
Es posible y adecuado realizar prevención en el marco escolar, tal como indica Pérez. ${ }^{28}$ Pero no sólo se trata de actuar desde los centros formales de educación. También se pueden desplegar iniciativas de tipo social, desde asociaciones, parroquias, ayuntamientos, centros de salud, movimientos de animación o educación social, etc.

\section{Factores de riesgo y factores protectores}

Existen factores de riesgo asociados a situaciones personales y sociales que predisponen al consumo y abuso del alcohol y de otras drogas (en el cuadro 2 se destacan algunos). Los factores protectores, por su parte, amortiguan o reducen la influencia de los primeros (cuadro 3).

\section{Actuaciones preventivas}

En el contexto de la adolescencia cumple un papel destacado el desarrollo de una autonomía emocional que incluye capacidades y competencias de autogestión personal, como la autoestima, la responsabilidad, el análisis crítico, la autonomía para la búsqueda de ayuda y recursos, la resiliencia, etc. ${ }^{29}$ La intervención educativa puede establecer estrategias dirigidas a la adquisición de una autonomía emocional que fortalecerá la capacidad del individuo para enfrentarse a situaciones que puedan atentar contra su salud a corto o mediano plazo.

La familia tiene una importancia capital en el desarrollo de las personas. Cuando en el seno familiar se utilizan pautas comunicativas positivas, ${ }^{30}$ muchos de los problemas que

Cuadro 2. Factores de riesgo

\begin{tabular}{|c|c|c|c|c|}
\hline Personales & Familiares & Escolares & $\begin{array}{c}\text { Relacionales } \\
\text { (grupo de iguales) }\end{array}$ & Socio-comunitarios \\
\hline $\begin{array}{l}\text { - Individualismo } \\
\text { - Imprudencia } \\
\text { - Escepticismo } \\
\text { - Inmediatismo } \\
\text { - Sistema de valores poco } \\
\text { claros } \\
\text { - Ausencia de normas y } \\
\text { límites } \\
\text { - Hedonismo } \\
\text { - Irresponsabilidad } \\
\text { - Actitud de evasión } \\
\text { - Dificultad en valorar el } \\
\text { esfuerzo } \\
\text { - Elección de conductas } \\
\text { de riego como reto a la } \\
\text { autoridad y autoafirma- } \\
\text { ción } \\
\text { - Bajo nivel de autoestima } \\
\text { - Bajo nivel de auto-control } \\
\text { emocional }\end{array}$ & $\begin{array}{l}\text { - Ausencia de afectividad } \\
\text { - Actitud permisiva y/o } \\
\text { desinteresada } \\
\text { - Falta de cohesión fami- } \\
\text { liar } \\
\text { - Pérdida figuras de } \\
\text { autoridad } \\
\text { - Sobreprotección } \\
\text { - Incoherencia educativa } \\
\text { - Ausencia de ocio fami- } \\
\text { liar compartido } \\
\text { - Falta de reconocimiento } \\
\text { y aceptación del hijo } \\
\text { - Aislamiento emocional } \\
\text { de los miembros }\end{array}$ & $\begin{array}{l}\text { - Problemas de adapta- } \\
\text { ción } \\
\text { - Desmotivación } \\
\text { - Bajo rendimiento acadé- } \\
\text { mico } \\
\text { - Bajo auto-concepto } \\
\text { escolar } \\
\text { - Ausencia de modelos de } \\
\text { referencia en el profeso- } \\
\text { rado } \\
\text { - Poca o nula integración } \\
\text { en el grupo de clase } \\
\text { - Problemas de integra- } \\
\text { ción de normas } \\
\text { - Bajo nivel de expectati- } \\
\text { vas } \\
\text { - Baja aceptación del } \\
\text { alumno/a }\end{array}$ & $\begin{array}{l}\text { - Excesiva dependencia } \\
\text { del grupo } \\
\text { - Inclusión en grupos } \\
\text { transgresores y con } \\
\text { actitud positiva hacia } \\
\text { las drogas } \\
\text { - Dificultad para relacio- } \\
\text { narse con otros grupos } \\
\text { no consumidores } \\
\text { - Escaso desarrollo de } \\
\text { habilidades sociales } \\
\text { - Exposición a modelos } \\
\text { consumidores } \\
\text { - Creación de oportunida- } \\
\text { des para el consumo }\end{array}$ & $\begin{array}{l}\text { - Situación socio-económi- } \\
\text { ca precaria } \\
\text { - Problemas de inclsión } \\
\text { social (colectivos } \\
\text { inmigrantes, minorías } \\
\text { étnicas) } \\
\text { - Desestructuración social } \\
\text { - Ausencia de apoyos } \\
\text { sociales } \\
\text { - Accesibilidad a las } \\
\text { sustancias } \\
\text { - Modelos consumistas } \\
\text { - Modelos culturales de } \\
\text { "descontrol y coloque" } \\
\text { - Escasez de ofertas alter- } \\
\text { nativas de ocio }\end{array}$ \\
\hline
\end{tabular}

Fuente: Extraído de Árbex. ${ }^{24}$ 
Cuadro 3. Factores protectores

\begin{tabular}{|c|c|c|c|c|}
\hline Personales & Familiares & Escolares & $\begin{array}{c}\text { Relacionales } \\
\text { (grupo de iguales) }\end{array}$ & Socio-comunitarios \\
\hline $\begin{array}{l}\text { - Altruismo } \\
\text { - Prudencia } \\
\text { - Proyección y planifica- } \\
\text { ción de futuro } \\
\text { - Clarificación en su esca- } \\
\text { la de valores } \\
\text { - Responsabilidad } \\
\text { - Actitud de solidaridad y } \\
\text { compromiso } \\
\text { - Valoración del esfuerzo } \\
\text { - Establecimiento y } \\
\text { cumplimiento de límites y } \\
\text { normas } \\
\text { - Adecuado nivel de } \\
\text { autoestima } \\
\text { - Autoconcepto positivo } \\
\text { - Autocontrol emocional y } \\
\text { conductual } \\
\text { - Habilidades sociales } \\
\text { (HHSS) }\end{array}$ & $\begin{array}{l}\text { - Ambiente familiar } \\
\text { afectivo y comunicación } \\
\text { positiva } \\
\text { - Actitud contraria al } \\
\text { consumo de drogas } \\
\text { - Límites y normas claras } \\
\text { - Cohesión familiar } \\
\text { - Supervisión y control } \\
\text { adecuados } \\
\text { - Roles ajustados y figuras } \\
\text { de autoridad de referen- } \\
\text { cia } \\
\text { - Exigencias adecuadas a } \\
\text { la edad } \\
\text { - Coherencia educativa } \\
\text { - Disfrute de ocio familiar } \\
\text { compartido } \\
\text { - Reconocimiento y acep- } \\
\text { tación del hijo/a } \\
\text { - Comunicación emocio- } \\
\text { nal de los miembros }\end{array}$ & $\begin{array}{l}\text { - Integración en la diná- } \\
\text { mica escolar } \\
\text { - Motivación } \\
\text { - Hábitos de estudio } \\
\text { - Adecuado rendimiento } \\
\text { - Autoconcepto escolar } \\
\text { positivo } \\
\text { - Modelos positivos de } \\
\text { referencia en el profeso- } \\
\text { rado } \\
\text { - Integración en el grupo } \\
\text { de clase } \\
\text { - Normas de aula } \\
\text { - Interés por lo escolar } \\
\text { - Aceptación del alumno/ } \\
\text { a en el grupo } \\
\text { - Experiencias de refuerzo } \\
\text { positivo ante el esfuerzo } \\
\text { - Valoración del alumnado } \\
\text { - Educación emocional } \\
\text { - Enseñanza de HHSS }\end{array}$ & $\begin{array}{l}\text { - Capacidad crítica y } \\
\text { desarrollo del criterio } \\
\text { propio } \\
\text { - Inclusión en grupos con } \\
\text { ausencia de conductas } \\
\text { desadaptativas } \\
\text { - Toma de decisiones } \\
\text { personales } \\
\text { - Modelos de amigos no } \\
\text { consumidores o consu- } \\
\text { mo moderado } \\
\text { - Desarrollo adecuado de } \\
\text { habilidades sociales } \\
\text { - Red amplia de amigos } \\
\text { - Participación en grupos } \\
\text { de carácter positivo } \\
\text { (asociaciones, clubes } \\
\text { deportivos, parroquias, } \\
\text { voluntariado...) }\end{array}$ & $\begin{array}{l}\text { - Organización social } \\
\text { adecuada } \\
\text { - Cohesión social en el } \\
\text { barrio } \\
\text { - Relaciones positivas de } \\
\text { vecindad } \\
\text { - Apoyos sociales y pro- } \\
\text { tección al menor } \\
\text { - Dificultades de acceso a } \\
\text { las sustancias } \\
\text { - Modelos valorados } \\
\text { culturalmente } \\
\text { - Recursos comunitarios } \\
\text { suficientes para los } \\
\text { menores } \\
\text { - Amplia oferta de disfrute } \\
\text { del ocio } \\
\text { - Integración e inclusión } \\
\text { social }\end{array}$ \\
\hline
\end{tabular}

Fuente: Extraído de Árbex. ${ }^{24}$

afectan a la adolescencia pueden ser paliados y resueltos satisfactoriamente, sin necesidad de recurrir a programas especializados. Sin embargo, en muchas ocasiones, las familias precisan orientación para mejorar las relaciones entre sus miembros, los conocimientos de los efectos perniciosos del alcohol y el autoanálisis sobre la conducta bebedora. De manera especial, cuando nos encontramos con grupos de alto riesgo, es fácil encontrar familiares que presentan problemas de alcoholismo, menudeo de droga, altos niveles de analfabetismo, etc. ${ }^{31}$

Para llevar a cabo acciones efectivas, es necesario detectar qué tipo de prevención se requiere y establecer los niveles del programa. Generalmente se requiere una intervención coordinada con los adolescentes y con sus familias. ${ }^{24}$ Es precisa una mayor implicación (conocimiento, dedicación y compromiso) y trabajo en red de toda la sociedad para potenciar los factores protectores y trabajar por conseguir una mayor calidad de vida. ${ }^{32}$ Son necesarias las intervenciones a largo plazo, en contextos dinámicos de mutua interacción e incorporadas en las actuaciones educativas, que nos ayuden a paliar los efectos derivados del abuso del alcohol y a promover empeños por la salud integral.

Pari passu la neurociencia y la educación han de mantener un diálogo fluido que permita conocer mejor las bases biológicas de la adicción ${ }^{33}$ y diseñar estrategias educativas eficaces $^{34}$ en el marco de un nexo consolidado entre educación y neurociencia.

\section{REFERENCIAS}

1. WHO. The second decade: improving adolescent health and development. Ginebra. 2001.

2. Sawyer SM, Afifi RA, Bearinger LH, Blakemore SJ et al. Adolescence: a foundation for future health. Lancet 2012;379:1630-1640.

3. Giedd JN. The teen brain: insights from neuroimaging. J Adolesc Health 2008;42:335-343.

4. Witt ED. Research on alcohol and adolescent brain development: opportunities and future directions. Alcohol 2010;44:119-124.

5. Guerri C, Pascual M. Mechanisms involved in the neurotoxic, cognitive, and neurobehavioral effects of alcohol consumption during adolescence. Alcohol 2010;44:15-26.

6. Garcia A, Benito J. Educación para la salud y elementos socioculturales de la alcoholización. Anales Pedagogía 2000;18:111-124.

7. Larrañaga E, Yubero S, Navarro R, Sánchez C. Factores sociales y educativos en el consumo de alcoholen jóvenes. Pedagogía social: Revista Interuniversitaria 2006;12:17-31.

8. DGPNSD. Informe de la comisión clínica htpp://www.pnsd.msc.es/ Categoría2/publica/pfd/InformeAlcohol.pdf. 2007.

9. ESTUDES.http://www.pnsd.msc.es/categoria2/observa/pdf/ESTUDES_2010.pdf. 2010.

10. Calafat A, Juan M, Becoña E, Castillo A et al. El consumo de alcohol en la lógica del botellón. Adicciones 2005;17:193-202.

11. Calafat A. El abuso de alcohol de los jóvenes en España. Adicciones 2007;19:217-224.

12. WHO. Evidence for the effectiveness and cost-effectiveness of interventions to reduce alcohol related harm. WHO Europe 2009.

13. Peoples RW, Li C, Weight FF. Lipid vs protein theories of alcohol action in the nervous system. Annu Rev Pharmacol Toxicol 1996;36:185-201.

14. Lovinger DM, Roberto M. Synaptic effects induced by alcohol. Curr Top Behav Neurosci 2013;13:31-86. 
15. Heilig M, Thorsell A, Sommer WH, Hansson AC et al. Translating the neuroscience of alcoholism into clinical treatments: from blocking the buzz to curing the blues. Neurosci Biobehav Rev 2010;35:334-344.

16. Gogtay N, Giedd JN, Lusk L, Hayashi KM et al. Dynamic mapping of human cortical development during childhood through early adulthood. Proc Natl Acad Sci USA 2004;101: 8174-8179.

17. Spear LP. Adolescent neurobehavioral characteristics, alcohol sensitivities, and intake: Setting the stage for alcohol use disorders? Child Dev Perspect 2011;5:231-238.

18. Grant BF, Stinson FS, Harford TC. Age at onset of alcohol use and DSM-IV alcohol abuse and dependence: a 12-year follow-up. J Subst Abuse 2001;13:493-504.

19. Hiller-Sturmhöfel S, Swartzwelder HS. Alcohol's effects on the adolescent brain. Alcohol Res and Health 2004/2005; 28: 213-221.

20. Pascual M, Blanco AM, Cauli O, Minarro J et al. Intermittent ethanol exposure induces inflammatory brain damage and causes long-term behavioural alterations in adolescent rats. Eur J Neurosci 2007;25:541-550.

21. Pascual M, Boix J, Felipo V, Guerri C. Repeated alcohol administration during adolescence causes changes in the mesolimbic dopaminergic and glutamatergic systems and promotes alcohol intake in the adult rat. J Neurochem 2009;108:920-931.

22. Maldonado-Devincci AM, Badanich KA, Kirstein CL. Alcohol during adolescence selectively alters immediate and long-term behavior and neurochemistry. Alcohol 2010;44:57-66.

23. Alling C, Rodriguez FD, Gustavsson L, Simonsson P. Continuous and intermittent exposure to ethanol: effect on NG 108-15 cell membrane phospholipids. Alcohol Alcohol 1991;(Sup)1:227-231.
24. Arbex C. Guía de intervención: Menores y consumos de drogas. Madrid: ADES; 2002.

25. Sanchiz ML. Modelos de orientación e intervención psicopedagógica. Castellón: Publicaciones Universitat Jaume I; 2008.

26. Pantoja L, Añaños F. Actuaciones socioeducativas con menores vulnerables en riesgo relacionados con las drogas. Reflexiones críticas. Pedagogía social: Revista Interuniversitaria 2010;17:109-122.

27. Bisquerra R. Modelos de orientación e intervención psicopedagógica. Barcelona: CISS-Praxis; 1998.

28. Pérez C (coord.). Prevención del consumo de alcohol y tabaco. Guía didáctica para el profesorado del primer ciclo de la ESO. Ministerio de Educación y Cultura. Ministerio de Sanidad y Consumo. 1999.

29. Bisquerra Alzina R, Pérez Escoda N. Las competencias emocionales. Revista Facultad Educació 2007;10:61-82.

30. Crespo JM. Bases para construir una comunicación positiva en la famila. Revista Investigación Educación 2011;9:91-98.

31. Sánchez JM. Un modelo educativo de atención al entorno familiar: los centros de día dirigidos a menores. Revista Investigación Educación 2011;9:223-235.

32. Del Bosque Garza J, Fernández Casares C, Alba Fuentes M, Díaz Negrete DB et al. Hacia una mejor respuesta ante el problema del abuso de bebidas con alcohol: el papel del Sector Salud. Salud Mental 2012;35:165-174.

33. Matus Ortega ME, Calva Nieves JC, Flores Zamora A, Leff Gelman $P$ et al. Las adicciones, la genómica y la proteómica. Salud Mental 2012;35:137-145.

34. Blakemore SJ, Bunge SA. At the nexus of neuroscience and education. Dev Cogn Neurosci 2012;2(Suppl 1): S1-S5.

Artículo sin conflicto de intereses 